\title{
Dynamic and Segmental Methods of Intensity Modulated Radiotherapy Delivery in Prostate Cancer - Analysis of Volumetric and Dosimetric Parameters.
}

\author{
Sateesh Kumar Gv ${ }^{1}$, Kesava Ramgopal A ${ }^{2}$ \\ ${ }^{1}$ (Department of Radiotherapy, Siddhartha Medical College, Vijayawada, India) \\ ${ }^{2}$ (Department of Radiation Oncology, NRI Medical College, Chinakakani, India)
}

\begin{abstract}
The use of intensity-modulated radiotherapy (IMRT) is now widely advocated for the treatment of prostate cancers, to increase the therapeutic ratio. IMRT is delivered with a conventional multileaf collimator $(M L C)$ either in dynamic mode (DMLC or sliding window) or in segmental mode (SMLC or step-and-shoot). This study aims to compare the volumetric and dosimetric parameters between the two methods of IMRT delivery.There was significant difference in favour of DMLC plans for Beam-on time (MU) for PTV46( $p=0.0001), \operatorname{PTV76}(p=0.0001)$, treatment delivery time $(p=0.0001)$, mean dose to normal healthy tissue $(p=0.047)$ and integral dose to normal healthy tissue $(p=0.028)$. There was no significant difference in Rectal mean dose $(p=0.858)$ and mean dose to bladder $(p=0.78)$, left femoral head $(p=0.885)$, right femoral head( $(p=0.887)$ for PTV46 and PTV76 in both the plans.Fluence dosimetric analysis showed DMLC plans were better than SMLC plans in fulfilling the gamma criteria (4\% $3 \mathrm{~mm})$.
\end{abstract}

Keywords : Intensity modulated radiotherapy, prostate cancer.

\section{Introduction}

There are several methods of radiotherapy delivery in prostate cancer and intensity-modulated radiotherapy (IMRT) is an important and latest technique in the management of prostate cancer. IMRT is better than over three-dimensional conformal radiotherapy (3DCRT) as it can conform the prescribed doses to the target volume while sparing the adjacent critical structures.[1] IMRT is delivered using a conventional MLC either as a segmental MLC(SMLC) or dynamic MLC(DMLC). The SMLC method involves delivery of radiation when MLC leaves are stationary, in the DMLC method MLC leaves are moving as the radiation is delivered.

The SMLC IMRT may be convenient to verify and is technically easier than a DMLC treatment.[2] A DMLC-based delivery requires more monitor units (MU) than an SMLC method, as the beam is kept on throughout the delivery of radiation[3]. Kry et al. have shown that depending on the treatment energy, IMRT using step and shoot requires 3.5-4.9 times more monitor units as compared to the conventional treatment.[4] Chui et al. have shown that a dynamic IMRT requires 20\% more MU as compared to static IMRT [5]. Alaei et al. have shown that SMLC on an average required 15\% lesser MUs than a DMLC with 15\% longer treatment time than an SMLC treatment.[6]

The choice of delivery technique, static or dynamic, has become a topic of debate due to substantial difference between the MUs required to deliver the same treatment. Increased MU is expected to increase the integral dose and the low-dose volume. As indicated by Hermanto et al. few studies have addressed the effect of IMRT on the volumes receiving very low doses, such as $5 \mathrm{~Gy}$, which may be more relevant to increasing the risk of second malignancies.[9] At higher doses the risk of inducing cancer will decrease due to dominant cell killing rather than cell mutations.[10] The differences between the MUs required to deliver the same treatment is attributed to the method of delivery. There are few studies that compared SMLC and DMLC in terms of dosimetric quality of the plans,[5],[6]. Other studies have analyzed the impact of number of beams, beam energy and the delivery technique on integral dose.[11],[12] These techniques have shown that integral dose has $<1 \%$ variation with number of beams and higher beam energies reduced the integral dose. No studies have compared DMLC and SMLC techniques with regard to integral dose and low-dose volume, which have a significant effect on the probability of radiation-induced malignancies. The main objective of this study is to evaluate the effect of the two IMRT delivery techniques DMLC and SMLC, planned using Eclipse (Varian Associates, Palo Alto, CA) to compare integral dose, mean rectal dose and mean bladder dose, beam-on time (MU or monitor units) and treatment delivery time and to compare the fluence dosimetric parameters (area gamma).

\section{Materials And Methods}

Ten cases of carcinoma of Prostate treated by IMRT were chosen for this study. Planning computed tomography (CT) images with a slice thickness of $2.5 \mathrm{~mm}$ were obtained for all patients while immobilized in 
treatment position. Target volumes and Organs at risk (OAR) were contoured using Eclipse TPS (Varian Associates, Palo Alto, CA). The gross tumor volume (GTV) included the radiologically apparent primary tumor and involved lymph nodes, CTV46 (clinical target volume) included the GTV and adjacent soft tissue and nodal regions. A dose of 76 Gy to GTV was prescribed. Seven equispaced coplanar beams were used to generate the IMRT treatment plans. All plans were made with $6 \mathrm{MV}$ photons. All plans were normalized at the isocenter and the prescription isodose surface was chosen such that at least $95 \%$ of the target volume receives the prescription dose.

The basic idea behind the sliding window method is to sweep the MLC leaf pairs of varying aperture sizes and speed over a field. The main advantage of this technique is that by properly choosing the speed and aperture sizes, it is possible to deliver any intensity profile. After the calculation of the optimal fluence by the Dose Volume Optimizer (DVO) that is necessary to achieve the desired dose distribution in the patient, the fluence profile is transferred to the LMC program to calculate the necessary leaf sequence pattern. While calculating the leaf sequence pattern the LMC takes into account the physical limitations of the MLC device (eg, minimum leaf gap that should be maintained to avoid collisions between leaves, maximum leaf travel, etc.), transmission and leakage through the leaves (ie, the rounded leaf-end transmission). While calculating the leaf sequence file, the LMC program optimizes the leaf sequence pattern iteratively to reduce the MU required to deliver the optimal fluence profile and also to keep the delivered and the optimal fluence profiles as close as possible. However, due to the inherent limitations of the delivery device, it is impossible to deliver exactly the same fluence profile as calculated by the DVO. Due to this reason, the delivered and the optimal fluence profiles are never the same. The final fluence or the fluence to be delivered is calculated by the LMC based on the final leaf sequence pattern. In order to predict the delivered dose distribution as accurately as possible, the dose calculation algorithm uses the actual fluence calculated by the LMC for the final dose calculation. For fields wider than the Effective Leaf Out of Carriage Distance (ELOC) (in other words, if the leaves are not able to reach the opposite edge of the field) the original field is split into subfields, which are narrower than the ELOC and a sliding window leaf motion plan is calculated for each subfield separately. Because it is practically impossible to accurately connect sharp edges of subfields, the subfields are given an overlapping area.

In the SMLC method, the continuous fluence profile is divided into equally spaced discrete intensity levels. Even though it is possible to divide a continuous profile into unequally spaced intensity levels, such strategies are less commonly used. The LMC in Eclipse treatment planning system equally divides the continuous fluence profile with a user-defined input for individual fields. The spatial resolution used in this study for SMLC is the same as that for DMLC. The framework used for leaf sequencing is almost the same for both DMLC and SMLC. The sliding Window algorithm is first applied to the optimal fluence calculated by the DVO and the leaf trajectories are sampled to a smaller number of segments. The number of segments to be sampled depends on the intensity level defined for that field and the maximum fluence value calculated for that field. For example, with 5 intensity levels and a maximum fluence (transmission factor) value of 2, we will have 10 sub-fields for that particular field. After this process, the sub-fields are fine-tuned by incorporating transmission and leakage through the leaves similar to DMLC. The Integral Dose (ID) was calculated as the mean dose times the volume of the structure as given in equation (1). To calculate the ID to the normal tissues outside the target volumes, all the target volumes were subtracted from the body volume (body minus target volumes) and referred to as the normal healthy tissue (NHT). The volume receiving 5 Gy (V5) or less than what was determined from the dose-volume histograms (DVH) calculated for the NHT. All the plans were later converted to deliver by the SMLC method, using the same optimal fluence generated for the sliding window technique.

The dose to target volumes and critical structures and integral dose were compared between the two plans. The mean rectal dose, mean bladder dose,mean dose to right and left femoral heads, beam-on time (MU or monitor units), treatment delivery time were compared. The fluence dosimetric parameters (area gamma) were also compared for different techniques. Statistical analyses were performed using a paired two-tailed Student $t$ test to determine whether there is any statistically significant difference in any of the parameters examined. Differences were considered statistically significant with $P \leq 0.05$.

\section{Results}

There was significant difference in favour of DMLC plans for beam-on time (MU) for PTV46( $\mathrm{p}=0.0001)$, PTV76( $\mathrm{p}=0.0001)$, treatment delivery time $(\mathrm{p}=0.0001)$, mean dose to normal healthy tissue $(\mathrm{p}=0.047)$ and integral dose to normal healthy tissue $(1)(\mathrm{p}=0.028)$.

There was no significant difference in mean rectal dose, mean bladder dose $(p=0.858,0.78)$ and mean dose to right femur(p=0.885), left femur(p=0.887) for PTV46 and PTV76 in both the plans.

Fluence dosimetric analysis showed DMLC plans were better than SMLC plans in fulfilling the gamma criteria (4\% 3mm).

\section{Discussion}


Integral dose or the total cumulative dose to normal untreated tissues is higher in IMRT as compared to conventional treatment.[13],[14]. In this study, we have compared the integral dose and the low-dose volume in the normal healthy tissues with SMLC and DMLC. DMLC plans increased the integral dose to NHT, significant difference was found in the volume receiving 5 Gy when compared with SMLC plans. SMLC with low intensity levels such as $5 \mathrm{~L}$ slightly degraded the dose uniformity in the target volumes. In DMLC the beam is continuously switched on, which increases the dose to the OARs due to transmission and leakage through the leaves.

There are proponents of both SMLC and DMLC techniques of IMRT delivery. Using a large number of intensity levels can make a SMLC method almost equivalent to DMLC. However, treatment time may be prohibitively long with such approach. Other advantages of SMLC include a simpler MLC control system and fewer monitoring units compared to dynamic treatments. Quality assurance for a SMLC plan is also easier than in DMLC. Nevertheless, a DMLC delivery can be faster than a SMLC delivery as the beam is continuously on.[5],[15]. Studies have shown that a leaf position error of $1 \mathrm{~mm}$ can result in $10 \%$ error in dose delivery, which demands extremely regular and stringent quality assurance for the entire delivery system.[16] This study is an attempt to-quantify the difference in the integral dose and low-dose volume with static and dynamic IMRT planned using Eclipse TPS delivered by a conventional linac with conventional MLC. The accuracy of the results in this study is highly dependent on the accuracy of the dose calculation models used in this study. The dose calculation models used in this study do not account for MLC scatter, collimator leakage and tongue-and-groove effect. The incorporation of these effects in the dose calculation models is likely to increase the difference in the results. Recently, Jang et al. have compared conventional dose calculation models with Monte Carlo methods, and have shown that conventional dose calculation models do not properly model the secondary radiation from MLC, which contributes significantly to the low-dose in the IMRT plans.[17] A more accurate dose calculation algorithm such as Monte Carlo method will provide more accurate results in such studies.

\section{Conclusion}

DMLC and SMLC methods of IMRT delivery showed similar volumetric parameters. DMLC method has an advantage in delivering the desired intensity profile with a higher degree of accuracy. The beam on time was higher by $15 \%$ for DMLC and treatment delivery time was higher for SMLC by $30 \%$. The choice of IMRT delivery depends on machine capability,quality assurance, beam-on time, delivery time and other clinical considerations.

\section{References}

[1]. Mohan R, Wu Q, Manning M, Schmidt-Ullrich R. Radiobiological considerations in the design of fractionation strategies for intensity-modulated radiation therapy of prostate cancers. Int J Radiat Oncol Biol Phys 2000;46:619-30.

[2]. Xia P, Ting JY, Orton CG. Segmental MLC is superior to dynamic MLC for IMRT delivery. Med Phys 2007;34:2673-5.

[3]. Mohan R, Arnfield M, Tong S,Wu Q, Siebers J. The impact of fluctuations in intensity patterns on the number of monitor units and the quality and accuracy of intensity modulated radiotherapy. Med Phys 2000;27:1226-37.

[4]. Kry SF, Salehpour M, Followill D, Marilyn S, Deborah AK, White RA, et al. The calculated risk of fatal secondary malignancies from intensity modulated radiation therapy. Int J Radiat Oncol Biol Phys 2005;62:1195-203.

[5]. Chui CS, Chan MF, Yorke E, Spirou S, Clifton LC. Delivery of intensity modulated radiotherapy with a conventional multileaf collimator: Comparison of dynamic and segmental methods. Med Phys 2001;28:2441-6

[6]. Alaei P, Higgins PD, Weaver R, Nguyen N. Comparison of dynamic and step-and shoot intensity-modulated radiation therapy planning and delivery. Med Dosim 2004;29:1-6.

[7]. Hall EJ. Intensity - modulated radiation therapy, protons and the risk of second cancers. Int J Radiat Oncol Biol Phys 2006;65:1-7

[8]. Hall EJ, Wuu CS. Radiation induced second cancers: The impact of 3D-CRT and IMRT. Int J Radiat Oncol Biol Phys 2003;56:838 .

[9]. Hermanto U, Frija EK, Lii MJ, Chang EL, Mahajan A, Woo SY. Intensity modulated radiotherapy (IMRT) and conventional three dimensional conformal radiotherapy for high grade gliomas: Does IMRT increase the integral dose to normal brain ? Int J Radiat Oncol Biol Phys 2007;67:1135-44.

[10]. Hall EJ, Wuu CS. Radiation-induced second cancers: The impact of 3D-CRT and IMRT. Int J Radiat Oncol Biol Phys 2003;56:838.

[11]. Aoyama H, Westerly DC, Mackie TR, Olivera GH, Bentzen SM, Patel RR, et al. Integral radiation dose to normal structures with conformal external beam radiation. Int J Radiat Oncol Biol Phys 2006;64:962-7.

[12]. D'souza WD, Rosen II. Nontumor integral dose variation in conventional radiotherapy treatment planning. Med Phys 2003;30:206571.

[13]. Bland MJ, Altman DG. Statistical method for assessing agreement between two methods of clinical measurement. Lancet 1986;1:307-10.

[14]. Pirzkall A, Carol M, Lohr F, Chang EL, Mahajan A, Woo SY. Comparison of intensity modulated radiotherapy with conventional conformal radiotherapy for complex shaped tumors. Int J Radiat Oncol Biol Phys 2007;67:1135-44.

[15]. Alaei P, Higgins PD, Weaver R, Nguyen N. Comparison of dynamic and step-and-shoot intensity-modulated radiation therapy planning and delivery. Med Dosim 2004;29:1-6.

[16]. LoSasso T, Chui CS, Ling CC. Physical and dosimetric aspects of a multileaf collimation system used in the dynamic mode for implementing intensity modulated radiotherapy. Med Phys 1998;25:1919-27.

[17]. Jang SY, Liu HH, Mohan R. Underestimation of low-dose radiation in treatment planning of intensity-modulated radiotherapy. Int J Radiat Oncol Biol Phys 2008;71:1537-46. 
Equation 1

[18]. Integral dose $=$ Mean dose $($ NHT $)$ X V5 (NHT); NHT = BODY - PTV 